\title{
Multiband Bandstop Filter using an I-Stub-Loaded Meandered Defected Microstrip Structure
}

\author{
Gyan Raj KOIRALA, Nam-Young KIM \\ RFIC Research Lab, Dept. of Electronic Engineering, Kwangwoon University, Nowon-gu, Seoul 139-701, Korea \\ thegrkoirala@gmail.com,nykim@kw.ac.kr \\ Manuscript received July 13, 2015
}

\begin{abstract}
This paper presents a compact multiband bandstop filter (BSF) that utilizes I-stubs embedded within a meandered defected microstrip structure (MDMS). The proposed design for obtaining a single stopband is analyzed by using a transmission line network model. On the basis of the single stopband structure, we designed and fabricated a dual-and tri-band bandstop filters operating at $2.5 / 6.78 \mathrm{GHz}$ and $1.98 / 5.6 / 7.78 \mathrm{GHz}$, respectively, thereby exploring the concept of generating as many stopbands by simply adding the same number of I-stubs. The proposed filter also features the possibility of tuning the resonance frequencies by varying the width of the I-stubs.
\end{abstract}

\section{Keywords}

Multiband, bandstop filter (BSF), defected microstrip structure (DMS), I-stub

\section{Introduction}

Multiband bandstop filters (BSFs) are of great importance as modern communication systems are designed for multiband operations, such as GSM $(0.9 / 1.8 \mathrm{GHz})$, WLAN (2.4/3.6/5.2 GHz), and WiMax (2.3/2.5/3.5 GHz) to name a few. Unlike bandpass filters (BPFs), the resonators of BSFs resonate at the stopband so that the transmission loss and group delay at the passband can be substantially improved [1], [2]. The unwanted signals existing at the different frequency levels can be suppressed by employing a compact and low cost multiband BSF. As a result, researchers are designing effective dual-, tri- and quad-band BSFs to suppress the multiple spurious frequency bands with a single BSF.

A number of techniques have been studied to achieve multiband BSFs over the years. A lowpass to bandstop frequency variable transformation [1], a coupling technique [3], stepped-impedance-resonator (SIR) [4], [5], defected ground structure (DGS) [6-9] and defected microstrip structure (DMS) [9-13], are some widely used techniques that are commonly employed for designing multiband BSFs. Size is the primary concern in using passive elements during lowpass to bandstop transformation, the ex- istence of spurious resonance is prominent in filters employing SIR, whereas filters using DGS have a major constraint in terms of radiation from the ground plane. In DMS, a uniform or non-uniform transmission line is etched away to make the resonator, but, unlike in DGS, the ground plane is kept intact. DMS possesses similar characteristics to DGS in terms of rejecting electromagnetic waves in some frequencies [12]. Additionally, DMS also features easier integration with planar microwave circuits and maintains less crosstalk compared to DGS [13].

In this paper, we have developed a new design concept of multiband BSF using a meandered DMS (MDMS) structure with compact size. In contrast to the literature presented in [12], [13], that uses two-section SIR, a trisection SIR (TSSIR) is embedded within the MDMS to provide an extra degree of freedom in the design of the filter circuit and to maintain the compactness. An extensive analysis of the proposed design is carried out for a single band bandstop filter (SBBSF) structure by using a transmission line network model with relevant mathematical and theoretical concepts. Dual- and tri-band bandstop filters are subsequently designed, fabricated and measured to validate the proposed design concept of generating the required number of stopbands by simply adding the same number of I-stubs. The individual tuning of the resonance frequencies by varying the width of the I-stubs is demonstrated to strengthen the implementation of the proposed filter for practical microwave applications operating between $1 \mathrm{GHz}$ to $10 \mathrm{GHz}$ frequency bands.

\section{Analysis of a Single-Band BSF}

The configuration of the proposed model for the generation of a single stopband is shown in Fig. 1(a). The design consists of a MDMS enclosing an I-stub connected by a high-impedance microstrip line on both sides, which can be represented by a simplified TSSIR model as shown in Fig. 1(b). The entire structure shown in Fig. 1(a) can be modelled in terms of a two-port network structure consisting of three subnetworks, as illustrated in Fig. 2. Subnetworks $N_{1}$ and $N_{3}$ are related to the meandered microstrip line structure with a total length, $L_{1}=L_{1 \mathrm{a}}+L_{1 \mathrm{~b}}+L_{1 \mathrm{c}}$ and impedance, $Z_{1}$, whereas, the subnetwork $N_{2}$ is related to the 


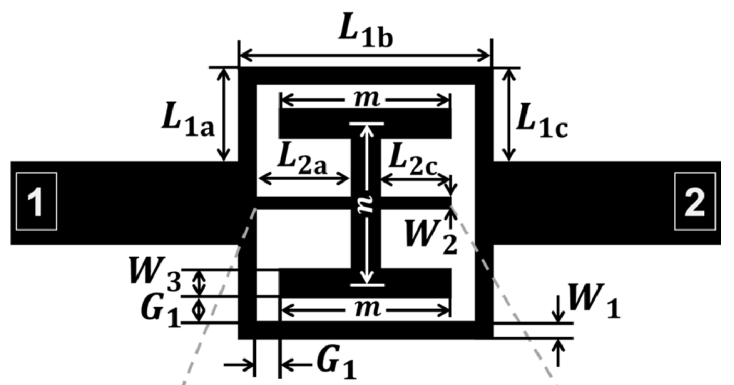

(a)

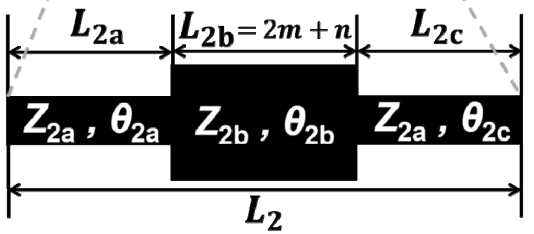

(b)

Fig. 1. The design model of the proposed filter. (a) The proposed SBBSF structure. (b) Simplified TSSIR structure of I-stub-loaded structure shown in (a).

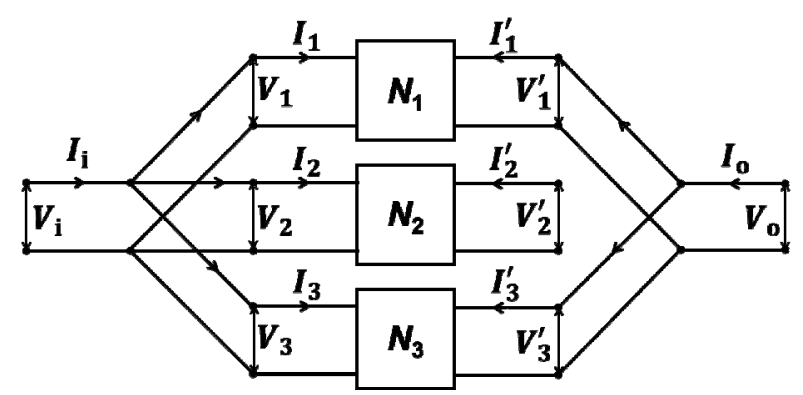

Fig. 2. The network model of the proposed SBBSF filter.

central open-ended TSSIR structure with a total length, $L_{2}=L_{2 \mathrm{a}}+L_{2 \mathrm{~b}}+L_{2 \mathrm{c}}$ and equivalent impedance, $Z_{2}$.

The lossless transmission line network model [14] can be applied in terms of $\mathrm{ABCD}$ parameters to find the current and voltage relationship of the subnetworks as follows

$$
\begin{aligned}
& {\left[\begin{array}{l}
V_{1} \\
I_{1}
\end{array}\right]=\left[\begin{array}{cc}
\cos \theta_{1} & j Z_{1} \sin \theta_{1} \\
j Y_{1} \sin \theta_{1} & \cos \theta_{1}
\end{array}\right]\left[\begin{array}{r}
V_{1}^{\prime} \\
-I_{1}^{\prime}
\end{array}\right],} \\
& {\left[\begin{array}{l}
V_{2} \\
I_{2}
\end{array}\right]=\left[\begin{array}{cc}
\cos \theta_{2} & j Z_{2} \sin \theta_{2} \\
j Y_{2} \sin \theta_{2} & \cos \theta_{2}
\end{array}\right]\left[\begin{array}{r}
V_{2}^{\prime} \\
-I_{2}^{\prime}
\end{array}\right],} \\
& {\left[\begin{array}{l}
V_{3} \\
I_{3}
\end{array}\right]=\left[\begin{array}{cc}
\cos \theta_{1} & j Z_{1} \sin \theta_{1} \\
j Y_{1} \sin \theta_{1} & \cos \theta_{1}
\end{array}\right]\left[\begin{array}{r}
V_{3}^{\prime} \\
-I_{3}^{\prime}
\end{array}\right]}
\end{aligned}
$$

where $Z_{i}=\left(1 / Y_{i}\right)$ for $i=1,2$ is the impedance of the respective sections and $\theta_{1}=\beta_{1} L_{1}$.

Furthermore, for an I-stub-loaded structure, the total electrical length, $\theta_{2}$, is given by

$$
\theta_{2}=\theta_{2 \mathrm{a}}+\theta_{2 \mathrm{~b}}+\theta_{2 \mathrm{c}}=\beta_{2 \mathrm{a}} L_{2 \mathrm{a}}+\beta_{2 \mathrm{~b}} L_{2 \mathrm{~b}}+\beta_{2 \mathrm{c}} L_{2 \mathrm{c}}
$$

where $\beta_{2 \mathrm{a}}, \beta_{2 \mathrm{~b}}$, and $\beta_{2 \mathrm{c}}$ are propagation constants of the TSSIR with physical lengths of $L_{2 \mathrm{a}}, L_{2 \mathrm{~b}}$, and $L_{2 \mathrm{c}}$, respectively.
The equivalent input impedance, $Z_{2}$, of the TSSIR with the series impedance line shown in Fig. 1(b) can be obtained from [15] as follows

$Z_{2}=\frac{j Z_{2 \mathrm{a}}\left[Z_{2 \mathrm{a}}\left(\tan \theta_{2 \mathrm{a}}+\tan \theta_{2 \mathrm{c}}\right)+Z_{2 \mathrm{~b}} \tan \theta_{2 \mathrm{~b}}\left(1-\tan \theta_{2 \mathrm{a}} \tan \theta_{2 \mathrm{c}}\right)\right]}{\left[Z_{2 \mathrm{a}}\left(1-\tan \theta_{2 \mathrm{a}} \tan \theta_{2 \mathrm{c}}\right)-Z_{2 \mathrm{~b}} \tan \theta_{2 \mathrm{~b}}\left(\tan \theta_{2 \mathrm{a}}+\tan \theta_{2 \mathrm{c}}\right)\right]}$

At the resonance condition, $Y_{2}=0$ such that

$$
R_{2} \tan \theta_{2 \mathrm{~b}} \tan \left(\theta_{2 \mathrm{a}}+\theta_{2 \mathrm{c}}\right)=1
$$

where $R_{2}=Z_{2 \mathrm{~b}} / Z_{2 \mathrm{a}}$ is the impedance ratio of the low-impedance section to the high-impedance section of the TSSIR.

Also from Fig. 2,

$$
\begin{gathered}
V_{\mathrm{i}}=V_{1}=V_{2}=V_{3}, \\
I_{\mathrm{i}}=I_{1}+I_{2}+I_{3}, \\
V_{\mathrm{o}}=V_{1}^{\prime}=V_{3}^{\prime}, \\
I_{\mathrm{o}}=I_{1}^{\prime}+I_{3}^{\prime} .
\end{gathered}
$$

By solving the above equations from (1) through (10), the input-output voltage-current relationship can be expressed in terms of the ABCD parameters as

$$
\left[\begin{array}{c}
V_{\mathrm{i}} \\
I_{\mathrm{i}}
\end{array}\right]=\left[\begin{array}{cc}
A & B \\
C & D
\end{array}\right]\left[\begin{array}{c}
V_{\mathrm{o}} \\
-I_{\mathrm{o}}
\end{array}\right]
$$

such that

$$
\left.\begin{array}{l}
A=\cos \theta_{1} \\
B=j 0.5 Z_{1} \sin \theta_{1} \\
C=j\left(2 Y_{1} \sin \theta_{1}+Y_{2} \cos \theta_{1} \tan \theta_{2}\right) \\
D=\cos \theta_{2}-0.5 Z_{1} Y_{2} \sin \theta_{1} \tan \theta_{2}
\end{array}\right\}
$$

The ABCD parameters in (12) can be transferred in terms of the transmission coefficient, $S_{21}$, as

$$
S_{21}=\frac{2}{A+B / Z_{0}+C Z_{0}+D}
$$

where $Z_{0}=50 \Omega$ is the port impedance.

The transmission zero occurs at the frequency where $\left|S_{21}\right|=0$; for which the necessary and sufficient condition is

$$
Z_{2} \cos \theta_{2}=0 \text {. }
$$

From (14),

$$
\theta_{2}=\left(n+\frac{1}{2}\right) \pi, n=0,1,2 \ldots
$$

It is worth mentioning that the theoretical analysis from (4) through (15) is derived for the simplified TSSIR model presented in Fig. 1(b). Therefore, several factors have to be accounted before applying the above analysis to the proposed SBBSF structure. First, an I-stub with symmetric open ends on both sides eventually increases the overall electrical field or the capacitance over there. Second, the discontinuity in the resonator structure plays sig- 
nificant role in the resonance. Third, the current distribution in the I-stub is not uniform as the current is mainly concentrated on the high impedance section, $Z_{2 \mathrm{a}}$, with an electrical length of $\theta_{2 \mathrm{a}}$. Hence, we only considered the

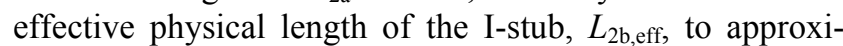
mate the resonance condition defined by (13). It is found that the effective physical and electrical length of the I-stub i.e. $L_{2 \mathrm{~b}, \text { eff }}$ and $\theta_{2 \mathrm{~b} \text {,eff }}$ could be closely approximated by taking two third of their total length i.e. $L_{2 \mathrm{~b}}$, and $\theta_{2 \mathrm{~b}}$, respectively.

Referring to (14) for obtaining transmission zero, we have designed a BSF resonator structure presented in Fig. 1(a) with $Z_{2 \mathrm{a}}=131.1 \Omega, \theta_{2 \mathrm{a}}=17.18^{\circ}, Z_{2 \mathrm{~b}}=90.53 \Omega$, $\theta_{2 \mathrm{~b}, \text { eff }}=61.9^{\circ}$, and $\theta_{2 \mathrm{c}}=12.89^{\circ}$ corresponding to the physical dimensions of $L_{1 \mathrm{a}}=L_{1 \mathrm{c}}=1.6 \mathrm{~mm}, L_{1 \mathrm{~b}}=4.3 \mathrm{~mm}$, $L_{2 \mathrm{a}}=1.6 \mathrm{~mm}, L_{2 \mathrm{~b}}=8.5 \mathrm{~mm}$ resulting $L_{2 \mathrm{~b}, \mathrm{eff}}=5.66 \mathrm{~mm}$, $L_{2 \mathrm{c}}=1.2 \mathrm{~mm}, W_{1}=0.3 \mathrm{~mm}, W_{2}=0.2 \mathrm{~mm}, W_{3}=0.5 \mathrm{~mm}$ and $G_{1}=0.4 \mathrm{~mm}$. Fig. 3 shows the simulation results of the proposed SBBSF. The simulation is carried out on a full wave simulation software, SONNET. The resonance frequency, thus obtained is $6.5 \mathrm{GHz}$ without any spurious resonance existing up to $25 \mathrm{GHz}$, i.e. nearly four times the fundamental resonance frequency.

The fundamental resonance frequency, $f_{0}$, can be defined as [16]

$$
f_{0}=\frac{c}{4 L_{2} \sqrt{\varepsilon_{\mathrm{re} 2}}}
$$

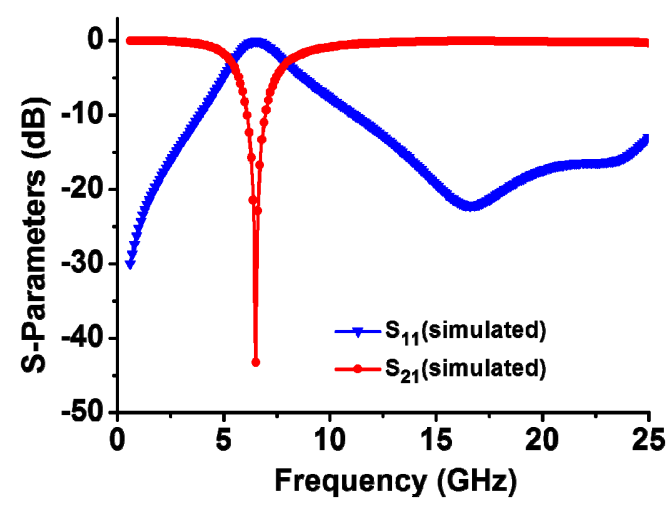

Fig. 3. Simulated results of the proposed SBBSF.

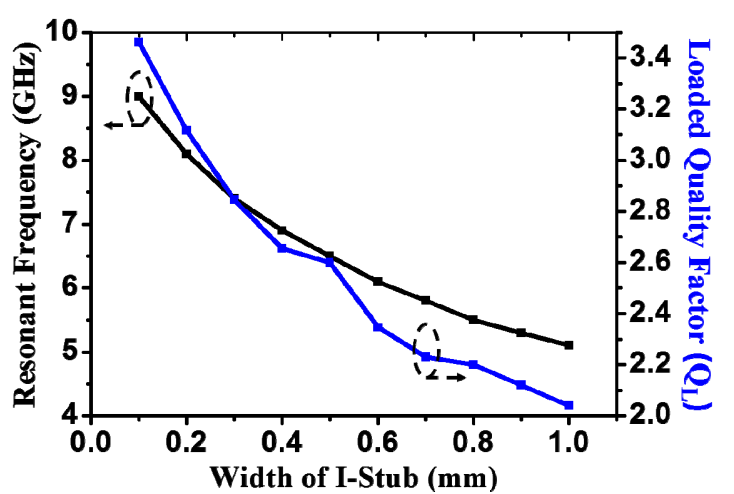

Fig. 4. Variation in the resonance frequency, $f_{0}$, and quality factor, $Q_{\mathrm{L}}$, of the SBBSF in accordance with the change in width of the I-stub, $W_{3}$. where $c=3 \times 10^{8} \mathrm{~m} / \mathrm{s}$ and $\varepsilon_{\mathrm{re} 2}$ is the average effective relative permittivity of the transmission line along with the length of the TSSIR. The resonance frequency, thus obtained from (16) having $L_{2}=L_{2 \mathrm{a}}+L_{2 \mathrm{~b}, \mathrm{eff}}+L_{2 \mathrm{c}}=8.46 \mathrm{~mm}$ and $\varepsilon_{\mathrm{re} 2}=1.99$ is $6.28 \mathrm{GHz}$, which is close to the EM simulated value of $6.5 \mathrm{GHz}$. Hence, it can be concluded that the resonance property of the proposed design depends upon the TSSIR.

Similarly, the characteristics of the resonance frequency, $f_{0}$, and loaded quality factor, $Q_{\mathrm{L}}$, are evaluated by varying the width of the I-stub as illustrated in Fig. 4. The loaded quality factor, $Q_{\mathrm{L}}$, can be closely approximated by the relation

$$
Q_{\mathrm{L}}=\frac{f_{0}}{B_{-3 \mathrm{~dB}}}
$$

where $B_{-3 \mathrm{~dB}}$ is the $-3 \mathrm{~dB}$ bandwidth of the stopband.

The graph in Fig. 4 reveals that there is a one-to-one correspondence between $f_{0}$ and $Q_{\mathrm{L}}$. The increment in the width of the I-stub, $W_{3}$, from $0.1 \mathrm{~mm}$ to $1.0 \mathrm{~mm}$ and keeping the gap, $G_{1}$, constant at $0.4 \mathrm{~mm}$ for all the sweeps of the structure results in the decrement of $f_{0}$ ranging from $9 \mathrm{GHz}$ to $5.1 \mathrm{GHz}$, whereas $Q_{\mathrm{L}}$ is decreased from 3.46 to 2.04. Thus, to maintain high $Q_{\mathrm{L}}$ of the proposed SBBSF, the width of the I-stub should be minimized under which $f_{0}$ will be increased.

\section{Design and Measurement of the Multiband BSF}

Based on the above analysis for SBBSF, dual- and triband BSFs were designed, fabricated and measured. Both the dual- and tri-band BSFs were fabricated on a Teflon substrate with a dielectric constant of $\varepsilon_{\mathrm{r}}=2.52$, a thickness of $h=0.504 \mathrm{~mm}$ and a loss tangent of 0.002 . Agilent $85052 \mathrm{D}$ VNA is used to measure the response of the fabricated design.

\subsection{Illustration of the Dual-Band BSF}

The fabricated design of the dual-band BSF (DBBSF) is shown in Fig. 5(a) along with the respective dimensions. The corresponding simulated and measured results are presented in Fig. 5(b). As compared to the SBBSF model presented in Fig. 1, DBBSF has an additional I-stub to generate the second stopband, thereby maintaining the compact size of only $18 \mathrm{~mm} \times 5.2 \mathrm{~mm}$, corresponding to $0.40 \lambda_{\mathrm{g}} \times 0.11 \lambda_{\mathrm{g}}$, where $\lambda_{\mathrm{g}}$ is calculated at $4.65 \mathrm{GHz}$, a mean value of first and second resonance frequencies. The resonance frequencies are measured at $2.52 \mathrm{GHz}$ and $6.78 \mathrm{GHz}$ with respective rejection levels of $30.54 \mathrm{~dB}$ and $29.20 \mathrm{~dB}$. Similarly, the $-3 \mathrm{~dB}$ fractional bandwidth (FBW) of the first and second stopband are measured to be $37.70 \%$ and $26.10 \%$, respectively.

The simulated current density profile of the proposed DBBSF at the two resonance frequencies of $2.5 \mathrm{GHz}$ and 


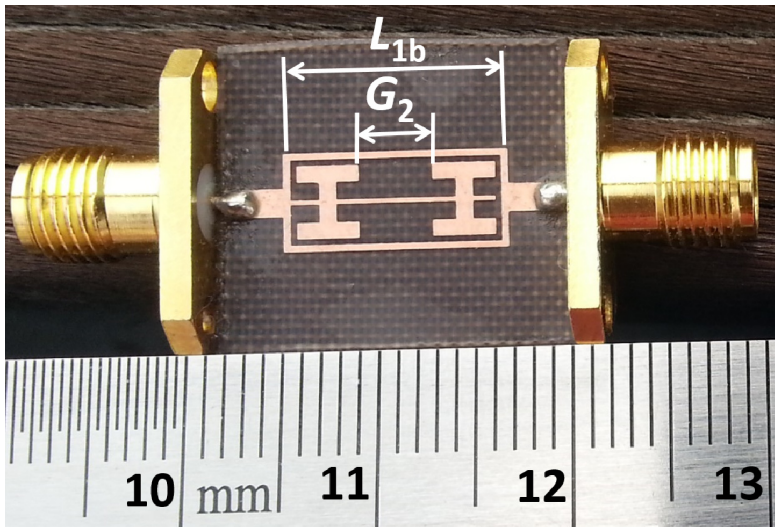

(a)

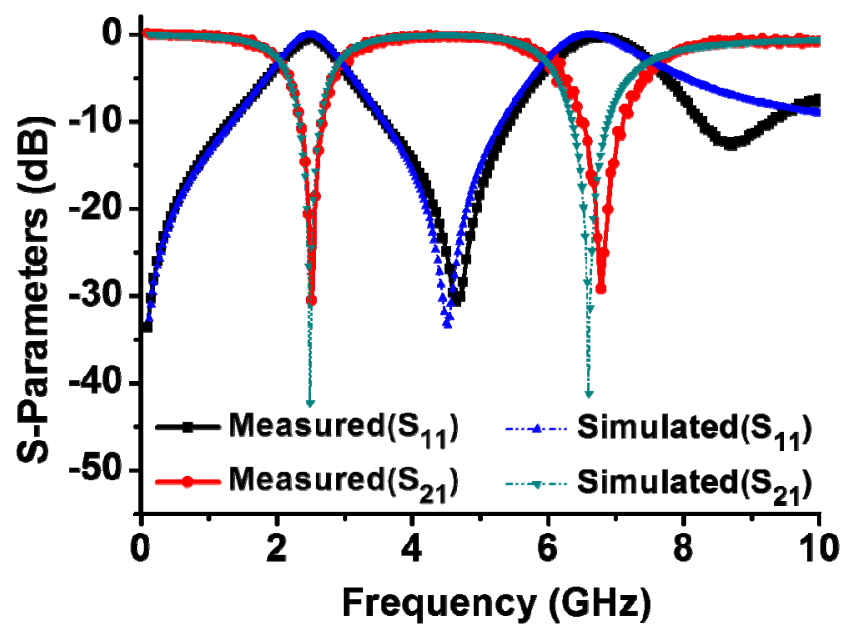

(b)

Fig. 5. Design and response of the proposed DBBSF. (a) Fabricated image of DBBSF with $L_{1 \mathrm{~b}}=11.75 \mathrm{~mm}$, $G_{2}=3.95 \mathrm{~mm}, W_{3}=0.8 \mathrm{~mm}$ and all other dimensions similar to those mentioned in Sec. 2. (b) Comparison between the simulated and measured S-parameters of the proposed DBBSF.

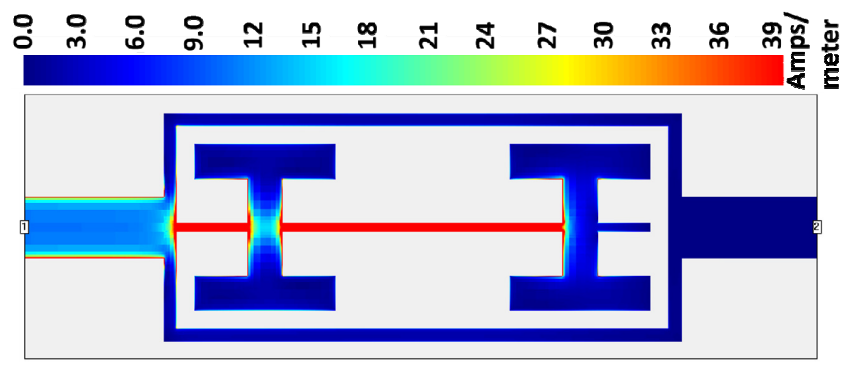

(a)@2.5 GHz

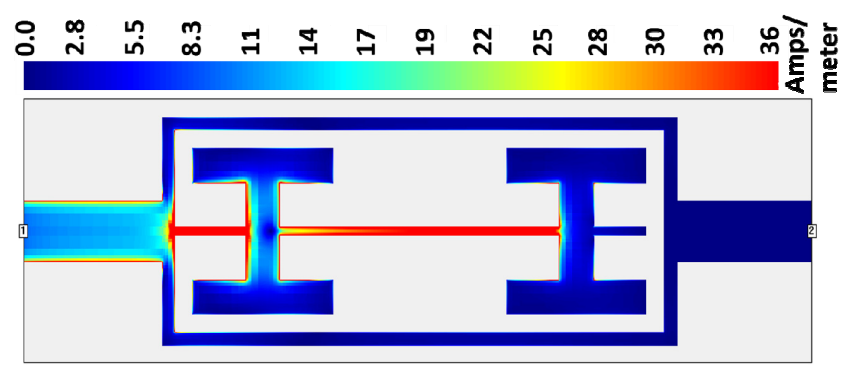

(b) @ $6.6 \mathrm{GHz}$

Fig. 6. Current distribution profile of the proposed DBBSF at the two resonance frequencies.
6.6 GHz are demonstrated in Fig. 6(a) and (b), respectively. The current distribution of both of the resonance frequencies seems to be concentrated on the high impedance stubs i.e. $L_{2 \mathrm{a}}, L_{2 \mathrm{c}}$ and $G_{2}$. Therefore, the I-stubs are responsible for the generation of the stopbands. The high current density value observed in Fig. 6(a) infers that any change in the second I-stub could have more effect on the first resonance frequency. Additionally, it can be attributed from Fig. 6(b) that the change in the width of the first Istub has significant shift at the second resonance frequency because the current density in the first I-stub is higher at the second resonance frequency.

The characteristics of the resonance frequencies were studied under three different conditions as shown in Fig. 7. The graph reveals that increasing in the width of the second I-stub, $W_{3}$, from $0.2 \mathrm{~mm}$ to $1.0 \mathrm{~mm}$ and keeping the width of the first I-stub constant at $0.8 \mathrm{~mm}$ resulted in the first resonance frequency decreasing notably from $3.35 \mathrm{GHz}$ to $2.25 \mathrm{GHz}$, whereas there was a significantly less impact on the second resonance frequency, as it was only decreased from 6.95 to $6.6 \mathrm{GHz}$ (expressed by case I). Conversely, the change in the width of the first I-stub, $W_{3}$, from $0.2 \mathrm{~mm}$ to $1.0 \mathrm{~mm}$ by fixing the width of the second I-stub at $0.8 \mathrm{~mm}$ had a significant effect on the second resonance frequency, which decreased from $9.15 \mathrm{GHz}$ to $6 \mathrm{GHz}$, but the first resonance frequency only decreased from $2.6 \mathrm{GHz}$ to $2.45 \mathrm{GHz}$ (expressed by case II). The results from case I and II signify that the increase in the width of the I-stub leads to the increase in the effective permittivity which approaches close to the dielectric constant of the substrate material and consequently reduces the resonance frequency. These outcomes also support the arguments drawn from the current distribution profile of the DBBSF shown in Fig. 6. Additionally, the change in the gap between the two I-stubs, $G_{2}$, shows a very minimal effect on both of the resonance frequencies, as the first and

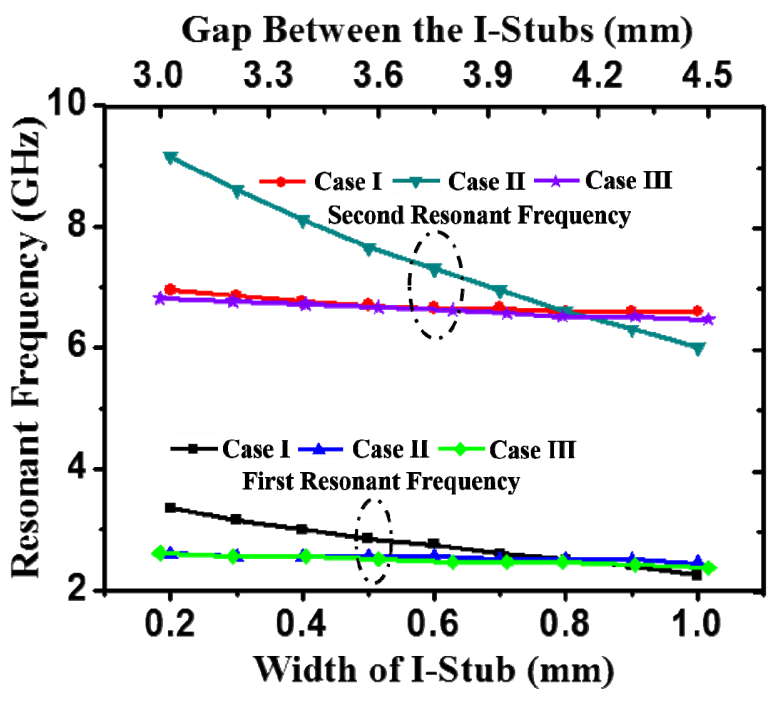

Fig. 7. Variation in resonance frequencies. Case I: Width of the second I-stub, $W_{3}$, is varied from 0.2 to $1.0 \mathrm{~mm}$. Case II: Width of the first I-stub, $W_{3}$, is varied from 0.2 to $1.0 \mathrm{~mm}$. Case III: Gap between the I-stubs, $G_{2}$, is varied between 3 and $4.5 \mathrm{~mm}$. 
second resonance frequencies were decreased from $2.65 \mathrm{GHz}$ to $2.4 \mathrm{GHz}$ and $6.85 \mathrm{GHz}$ to $6.5 \mathrm{GHz}$, respectively (expressed by case III). Therefore, it is possible to independently tune the resonance frequencies of the proposed BSF by varying the widths of the I-stubs.

\subsection{Illustration of the Tri-Band BSF}

The tri-band BSF (TBBSF) was designed on the basis of the aforementioned design process, and its fabricated image is shown in Fig. 8(a) along with the respective dimensions. The TBBSF utilizes three I-stubs to generate three stopbands with a constant gap of $G_{2}$ between two consecutive I-stubs. The simulated and measured results of the fabricated TBBSF are presented in Fig. 8(b). The overall size of the TBBSF is $20 \mathrm{~mm} \times 5.2 \mathrm{~mm}$, corresponding to $0.49 \lambda_{\mathrm{g}} \times 0.13 \lambda_{\mathrm{g}}$, where $\lambda_{\mathrm{g}}$ is calculated at $5.12 \mathrm{GHz}$, a mean value of the first, second and third resonance frequencies. The TBBSF generates resonance frequencies at $1.98 \mathrm{GHz}, 5.6 \mathrm{GHz}$ and $7.78 \mathrm{GHz}$, resulting their respective rejection levels of $28.71 \mathrm{~dB}, 25.17 \mathrm{~dB}$ and $25.02 \mathrm{~dB}$. The fractional bandwidth measured at a $-3 \mathrm{~dB}$ insertion loss is found to be $41.92 \%, 14.28 \%$ and $14.01 \%$, respectively,

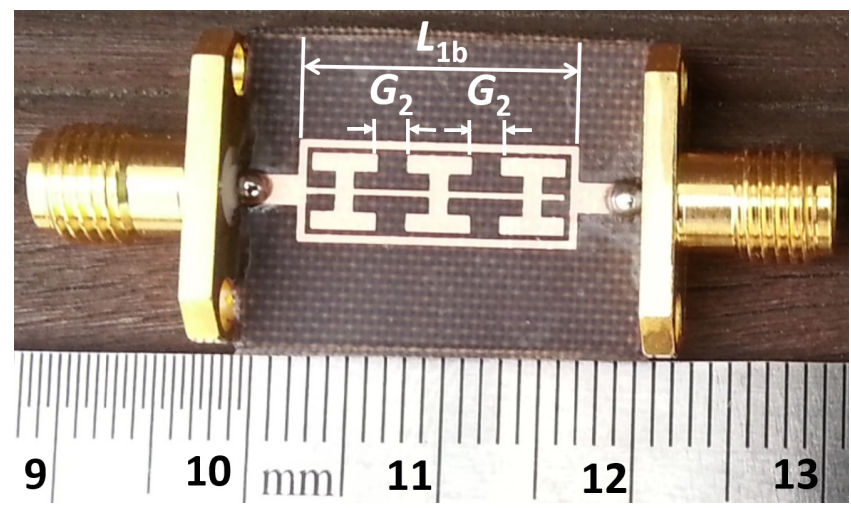

(a)

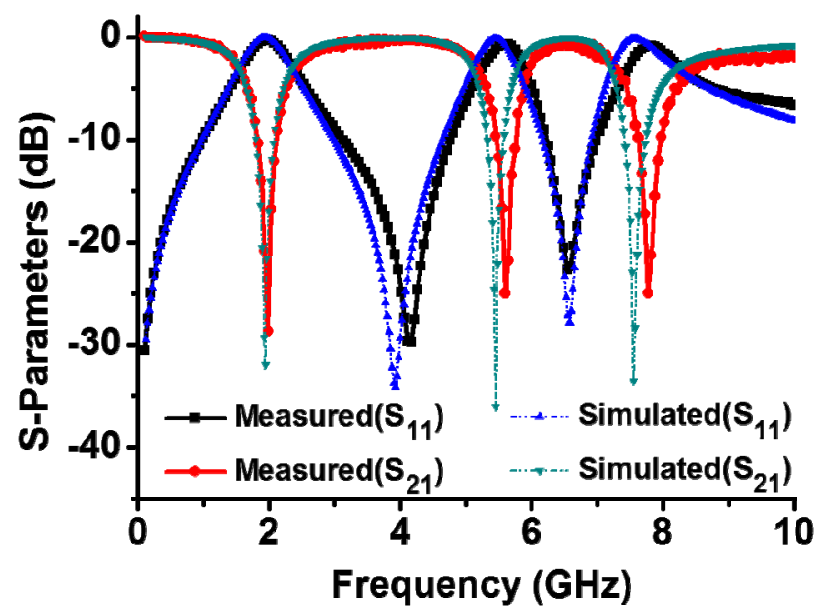

(b)

Fig. 8. Design and response of the proposed TBBSF (a) Fabricated image of the TBBSF with $L_{1 \mathrm{~b}}=$ $14.27 \mathrm{~mm}, G_{2}=1.64 \mathrm{~mm}, W_{3}=0.8 \mathrm{~mm}$ and all other dimensions similar to those mentioned in Sec. 2 . (b) Comparison between the simulated and measured S-parameters of the proposed TBBSF. for the first, second and third stopbands. Tuning of the TBBSF can be achieved by varying the widths of the Istubs following a process similar to that explained in the case of the DBBSF.

\section{Conclusion}

A promising approach for the design of microstrip multiband BSFs with a very simple structure and compact size is demonstrated. By adding the I-stubs, the required number of stopbands can be generated with minimal increase in the size of the filter. The reported works also show that the individual tuning of the stopbands is possible by varying the widths of the I-stubs. With a simple design process, resulting good agreement between simulated and measured results, individually adjusted stopbands, and having the capability of integrating with the other devices, the proposed filter shows great potential for multiband microwave applications, such as WLAN and WiMax technologies.

\section{Acknowledgments}

This work was supported by the National Research Foundation of Korea (NRF) grant funded by the Korean Government (MSIP), No. 2013-067321, and the grant supported by the Korean Government (MEST), No. 2012R1A1A2004366. This work was also supported by the research grant from Kwangwoon University in 2015.

\section{References}

[1] UCHIDA, H., KAMINO, H., TOTANI, K., et al. Dual-bandrejection filter for distortion reduction in RF transmitters. IEEE Transactions on Microwave Theory and Techniques, 2004, vol. 52, no. 11 , p. 2550-2556. DOI: 10.1109/TMTT.2004.837161

[2] SHRESTHA, B., KIM, N.Y. Slot meander spurline bandstop filter using integrated passive device technology. Microwave and Optical Technology Letters, 2014, vol. 57, no. 1, p. 168-172. DOI: 10.1002/mop. 28806

[3] SHAMAN, H., HONG, J.S. Wideband bandstop filter with crosscoupling. IEEE Transactions on Microwave Theory and Techniques, 2007, vol. 55, no. 8, p. 1780-1785. DOI: 10.1109/TMTT.2007.901600

[4] DHAKAL, R., KIM, N.Y. A compact symmetric microstrip filter based on a rectangular meandered-line stepped impedance resonator with a tri-band bandstop response. The Scientific World Journal, 2013. DOI: 10.1155/2013/457693

[5] ADHIKARI, K.K., KIM, N.Y. A miniaturized Quad-band bandstop filter using shunt connected, T-shaped stub-loaded steppedimpedance resonators. Microwave and Optical Technology Letters, 2015 , vol. 57, no. 5, p. 1129-1132. DOI: $10.1002 /$ mop. 29046

[6] WOO, D.J., LEE, T.K., LEE, J.W., et al. Novel U-slot and V-slot DGSs for bandstop filter with improved $\mathrm{Q}$ factor. IEEE 
Transactions on Microwave Theory and Techniques, 2006, vol. 54, no. 6, p. 2840-2847. DOI: 10.1109/TMTT.2006.875450

[7] CHEN, F.C., ZHANG, N.Y., ZHANG, P.S., et al. Design of ultrawideband bandstop filter using defected ground structure. Electronics Letters, 2013, vol. 49, no. 16, p. 1010-1011. DOI: 10.1049/el.2013.1541

[8] BARAL, R.N., SINGHAL, P.K. Recent techniques in design and implementation of microwave planar filters. Radioengineering, 2008 , vol. 17 , no. 4, p. 65-73.

[9] WANG, J., NING, H., XIONG, Q., et al. A novel miniaturized dual-band bandstop filter using dual-plane defected microstrip structures. Progress in Electromagnetics Research., 2013, vol. 134, p. 397-417.

[10] LA, D.S., HAN, W.H., ZHANG, J.L. Compact band-stop filters using $\pi$-shape DGS and $\pi$-shape DMS. Microwave and Optical Technology Letters, 2014, vol. 56, no. 11, p. 2504-2507. DOI: 10.1002/mop. 28625

[11] XIAO, J.K., ZHU, Y.F., LI, Y. Controllable miniature tri-band bandpass filter using defected microstrip structure. Electronics Letters, 2013, vol. 50, no. 21, p. 1534-1536. DOI: 10.1049/el.2014.1432

[12] XIANG, Q.Y., FENG, Q.Y., HUANG, X.G. Bandstop filter based on complementary split ring resonators defected microstrip structure. Journal of Electromagnetic Waves and Applications, 2011, vol. 25, p. 1895-1908. DOI: 10.1163/156939311797453999

[13] XIAO, J. K., ZHU, Y. F. Multi-band bandstop filter using inner Tshaped defected microstrip structure (DMS). AEU-International Journal of Electronics and Communications, 2014, vol. 68, no. 2, p. 90-96. DOI: 10.1016/j.aeue.2013.07.002

[14] HONG, J.S., LANCASTER, M.J. Microstrip Filters for RF/Microwave Applications. $1^{\text {st }}$ ed., New York: Wiley, 2001. ISBN: 0-471-22161-9

[15] ZHANG, H., CHEN, K.J. A tri-section stepped-impedance resonator for cross-coupled bandpass filters. IEEE Microwave and Wireless Components Letters, 2005, vol. 15, no. 6, p. 401-403. DOI: 10.1109/LMWC.2005.850475

[16] WANG, J., NING, H., XIONG, Q., et al. A compact narrow-band bandstop filter using spiral-shaped defected microstrip structure. Radioengineering, 2014, vol. 23, no. 1, p. 209-213.

\section{About the Authors ...}

Gyan Raj KOIRALA was born in Nepal in 1987. He received the Bachelor of Engineering degree in Electronics and Communication Engineering in 2010 from Tribhuvan University, Nepal. Following his Bachelor's Degree, Mr. Koirala served as a lecturer in Kathmandu Engineering College, Tribhuvan University, Nepal before joining Integrated M. S and Ph.D. Degree in Kwangwoon University, Seoul, Korea in 2014. His current research includes the design of RF/microwave devices, integrated passive device technology and RF biosensors.

Nam-Young KIM is the corresponding author for this manuscript. He was born in Korea in 1960. He has received two Masters and two Ph.D. degrees from State University of New York at Buffalo and Midwest University: the M.S. and the Ph.D. in Electronic Engineering. The other degree is M.Div and D.C.E in Theology. He was a research scientist for CEEM at SUNY at Buffalo in 1994. After completing his research at CEEM at SUNY at Buffalo, he joined the Department of Electronic Engineering of Kwangwoon University as an assistant professor in 1994. His main research focus is RFIC devices, ICs and systems that use wireless application techniques in order to develop high-speed structures in GaAs, $\mathrm{Si}$, and other materials. The founder of the RFIC Center, Dr. Kim also serves as director for the Fusion Technology Center of RF and Bio-related research. His RFIC research center was honored and sponsored as an ITRC (Information Technology Research Center) by the Ministry of Communication and Information (MIC) of Korea, where he has been acting Director of the RFIC since 2000. During his career, he has published 25 books, 171 refereed SCI journal papers and 304 refereed conference papers. Dr. Kim has been issued more than 60 patents and 96 semiconductor design patents. His research fields are the areas of RF semiconductor devices, RFICs and MMICs, LEDs and Biosensor applications. 